\title{
Amizades internacionais de universitários brasileiros: um estudo exploratório
}

\author{
Agnaldo Garcia \\ Universidade Federal do Espírito Santo
}

\begin{abstract}
Resumo
Amizades internacionais, entre pessoas de diferentes nacionalidades e culturas, ainda são pouco conhecidas. Este artigo investigou as amizades internacionais de estudantes universitários brasileiros utilizando um questionário contendo questões fechadas e abertas, com a participação de 120 universitários de ambos os sexos. Foram investigados: a rede internacional de amigos, a comunicação com esses amigos, início, interesses comuns e dificuldades nas amizades e a relação com o país do amigo. Ao todo foram citados 331 amigos de 59 países. Estas amizades geralmente se iniciaram por contato pessoal e eram mantidas pela Internet. Os dados foram discutidos à luz das propostas de Robert Hinde. Sugere-se a possibilidade das amizades internacionais servirem de base para a ampliação da cooperação cultural e científica entre diferentes países.
\end{abstract}

Palavras-chave: amizade; estudantes universitários; relações interpessoais

\begin{abstract}
International friendships of Brazilian college students: an exploratory investigation. International friendships involving individuals from different nations and cultures have not been widely investigated. This paper investigated the international friendships of Brazilian college students with the participation of 120 students, 46 males and 74 females, who answered a questionnaire with open and closed questions on international friends network, communication with friends, friendship beginnings, shared interests and difficulties in friendships and the perception of the friend's country. In sum, 331 friends from 59 countries have been mentioned. Friendships usually started by personal contact but they were maintained by Internet. The theoretical framework proposed by Hinde was used to discuss and interpret data. It is suggested that international friendships should serve as basis for fostering cultural and scientific cooperation among countries.
\end{abstract}

Keywords: friendship; college students; interpersonal relations

$\mathrm{O}$ papel da educação para o desenvolvimento de amizades internacionais entre os povos foi destacado na Declaração Universal dos Direitos do Homem: "a instrução promoverá a compreensão, a tolerância e a amizade entre todas as nações e grupos raciais ou religiosos" (Organização das Nações Unidas, 1948, Artigo 26). Nesse mesmo sentido, De Zafra (1953) enfatizou a importância dos laços pessoais de amizade entre pessoas de diferentes países, baseando-se na premissa de que relações amistosas entre países se baseiam nas relações amistosas entre seus cidadãos.

Passado mais de meio século das declarações acima, o conhecimento sobre amizades internacionais ou interculturais entre pessoas de diferentes países e culturas, ainda é uma área pouco investigada, especialmente no continente sul-americano. Conhecer melhor como pessoas pertencentes a países e culturas diferentes se tornam amigas e assim permanecem representa uma contribuição para a compreensão dos fatores que permitem sua integração social e cultural.
As amizades interculturais ou internacionais têm sido investigadas principalmente entre estudantes universitários, geralmente quando se encontram fora de seu país de origem (Bektas, 2008; Ward \& Margoret, 2004) sendo menos frequentes estudos sobre universitários do país anfitrião que entram em contato com esses estudantes estrangeiros (Ward, 2001). Estes estudos têm procurado identificar as limitações e dificuldades encontradas por esses estudantes para fazer amigos com pessoas do país anfitrião, tendendo a fazê-lo com outros estudantes do mesmo país de origem (Bailey, 2006; Brown, 2009a; Cushner \& Karim, 2004; Pandian, 2008; Sawir, Marginson, Deumert, Nyland, \& Ramia, 2008; UKCOSA, 2004). Enquanto as amizades com pessoas do país anfitrião contribuem para a aprendizagem do idioma e da cultura local, as com pessoas de mesma nacionalidade ajudam a reduzir o estresse e a solidão (Ward, 2001; Ward, Bochner, \& Furnham, 2001) e obter apoio instrumental (Brown, 2008), emocional, social e espiritual (Maundeni, 2001; Zhao \& Wildemeersch, 2008). 
A formação de amizades internacionais deve muito à internacionalização da educação superior (Alfantookh \& Bakry, 2008; Kreber, 2009), mas os fatores envolvidos em seu aprofundamento ainda são pouco investigados, como é o caso da auto-revelação (Chen, 2006). As diferenças culturais têm mostrado um papel ambivalente nessas amizades. Por vezes são consideradas como fatores limitantes, dificultando o estabelecimento e o desenvolvimento de relacionamentos interculturais, como no caso de conflito entre diferentes conceitos de amigo em diferentes culturas (Gareis, 2000). Em outras investigações, as diferenças culturais são vistas de modo positivo, graças a fatores como receptividade a outras culturas, orientação e empatia transcultural (Kudo \& Simkin, 2003). A exploração de diferentes culturas e idiomas nas amizades interculturais também foi apontada como um aspecto positivo dessas amizades, ao lado da assistência prestada (Lee, 2006).

No Brasil, poucos trabalhos investigando aspectos da vida desses estudantes, por vezes, fazem menção a suas dificuldades em fazer amigos brasileiros (Desidério, 2006), indicam a amizade com brasileiros como um fator positivo (Subuhana, 2009), ou incluem itens sobre amizade em avaliações da adaptação e satisfação com o contexto de vida no Brasil (Andrade \& Teixeira, 2009). Apesar da possibilidade de contato direto com pessoas de outros países e culturas, os resultados obtidos com os estudantes universitários internacionais, em termos do aumento da tolerância e da aproximação cultural entre os povos ao retornarem, tem sido considerado pequeno (Cushner \& Karim, 2004), assim como a melhoria nas habilidades transculturais (Ward, 2001) e na competência intercultural (Brown, 2009b).

A possibilidade de amizades internacionais com um contato pessoal menos intenso, também representa um importante ponto de reflexão frente a visões clássicas da amizade, como é o caso de Aristóteles. Segundo Ortega (2002), para Aristóteles, a amizade perfeita estaria baseada na virtude e o amigo seria amado por si mesmo, caracterizando-se por sua permanência no tempo e sua raridade. De acordo com Rocha (2006), a convivência seria indispensável para a amizade perfeita de acordo com Aristóteles, pois trata-se de "viver junto" e "viver na intimidade", quando os amigos dão e recebem, ajudam e são ajudados, amam e são amados.

Conhecer melhor amizades interculturais ou internacionais de universitários brasileiros pode fornecer informações importantes para promover a aproximação entre estudantes de países e culturas diferentes, com reflexos para a cooperação social, cultural e científica internacional.

Do ponto de vista teórico, a pesquisa se baseia na literatura sobre amizades interculturais ou internacionais e na obra de Robert Hinde (1997) sobre relacionamento interpessoal. Hinde, com base na Etologia Clássica, propõe alguns princípios para a construção de uma ciência dos relacionamentos, com ênfase na descrição e na análise de diferentes níveis de complexidade e suas relações dialéticas, partindo de interações e passando para relacionamentos, grupos e a sociedade. Ainda aponta a influência do ambiente físico e das estruturas socioculturais sobre os relacionamentos.

A existência de amizades internacionais envolvendo universitários brasileiros, observada em estudos anteriores, deu origem a questões relativas a aspectos que seriam relevantes nessas amizades. Em primeiro lugar, era necessário conhecer quem seriam esses amigos em termos de sua nacionalidade, uma vez que pertencer a diferentes países é a propriedade que torna essas amizades internacionais. Pertencer a diferentes países parecia gerar uma série de características que seriam próprias dessas amizades, como falar diferentes idiomas, apresentar um contato pessoal menor e enfrentar possíveis diferenças culturais. Assim, buscou-se investigar como seria possível a comunicação entre esses amigos, não somente pelas possíveis diferenças de idioma, mas também pelos meios de comunicação empregados, devido a dificuldades para manter contato pessoal permanente. Também seria importante conhecer como essas amizades teriam iniciado, uma vez que pertencer a diferentes países parecia ser algo que dificulta o contato entre as pessoas. Frente a diferenças culturais, também era relevante investigar a possibilidade de compartilhar interesses com pessoas de outras culturas e países, além de ter acesso às dificuldades que poderiam ser geradas por essa situação particular e se amizades pessoais poderiam despertar interesse pelo país do amigo, o que poderia servir de motivação para uma maior aproximação em relação a esse país, inclusive gerando algum tipo de cooperação cultural.

O objetivo desta pesquisa foi investigar a rede de amigos estrangeiros de universitários brasileiros e aspectos que seriam particularmente relevantes nessas amizades, face ao fato desses amigos pertencerem a outro país e cultura, como a comunicação com esses amigos, o início dessas amizades, os interesses compartilhados, as dificuldades enfrentadas nessas amizades e a relação com o país do amigo. A expressão "amizade internacional", neste trabalho, significa um relacionamento entre pessoas de diferentes nacionalidades, mantendo uma ligação importante com o país de origem, como é o caso dos estudantes internacionais.

\section{Método}

\section{Participantes}

Participaram da pesquisa 120 estudantes universitários brasileiros, sendo 74 do sexo feminino e 46 do sexo masculino. A idade dos participantes variou de 18 a 40 anos, com a seguinte distribuição: 18 anos (6), 19(14), 20(15), 21(16), 22(24), 23(13), 24(12), 25(9), 26(1), 27(4), 32(3), 36(1), 37(1) e 40 (1). Os estudantes eram provenientes de oito estados brasileiros: ES (89), SC (11), PA (10), PE (5), AM (2), PR (1), BA (1) e MG (1). Dos participantes, 71 já haviam viajado para o exterior e 49 não.

\section{Procedimento de coleta de dados}

Estudantes universitários de graduação com amigos estrangeiros foram convidados a participar da pesquisa. Ao aceitar o convite, os mesmos assinavam um termo de consentimento para participação na pesquisa e respondiam a um questionário composto por questões abertas e fechadas, elaboradas com base em pesquisas anteriores sobre o tema. $\mathrm{O}$ instrumento informava que amizades internacionais podiam ser com estrangeiros residindo no exterior ou no Brasil (neste caso, desde que há menos de três anos). Inicialmente, foram 
solicitadas informações sociodemográficas do participante, como idade e local de nascimento, além de informações sobre experiência internacional dos mesmos (viagens para o exterior). A seguir, os participantes deviam preencher uma tabela com até cinco amigos estrangeiros, informando nacionalidade do amigo e o idioma da comunicação. Quatro questões fechadas procuraram investigar a principal forma de comunicação com cada amigo indicado (pessoal, por telefone, por Internet ou outra, que deviam especificar), a forma como se deu o início de cada amizade (contato pessoal no exterior, contato pessoal no Brasil, contato online ou outra, que deviam especificar), os interesses comuns (lazer, ciências, cinema, música, turismo, literatura, esportes, artes, religião e assuntos familiares ou outro, que deviam especificar) e os interesses em relação ao país do melhor amigo estrangeiro (visitar o país como turista, estudar nesse país, trabalhar nesse país, residir nesse país ou nenhum interesse em particular). Para investigar as dificuldades percebidas foi proposta a seguinte questão aberta: "Que dificuldades encontra nessa amizade? Favor comentar”.

\section{Procedimento de análise de dados}

Os dados referentes à rede internacional de amigos, à comunicação com amigos internacionais, início da amizade, interesses comuns e relação com o país do amigo foram apresentadas quantitativamente, de forma descritiva. As respostas referentes às dificuldades com amizades internacionais foram analisadas por análise de conteúdo, com base em Bardin (1977). O conteúdo foi organizado em quatro categorias temáticas, de modo indutivo. Os temas presentes nas respostas foram organizados nas seguintes categorias: ausência física ou distância, diferenças culturais, conteúdo da relação e características individuais. A descrição de cada grupo temático foi apresentada nos resultados. Os dados foram analisados de modo qualitativo. No decorrer dos resultados, os números entre parênteses indicaram qual o participante (de 1 a 120) relatou o item em questão. Por vezes, foram inseridas as palavras do próprio participante como exemplos de cada caso.

\section{Resultados}

Os resultados foram apresentados em cinco itens. Primeiramente, foram apresentados dados referentes à composição da rede internacional de amigos com base na nacionalidade desses amigos. Tendo em vista as possíveis dificuldades devido a diferenças culturais, de idioma e falta de contato pessoal, o item seguinte trata da comunicação com esses amigos internacionais, quanto aos meios e idioma de comunicação, uma vez que esta é fundamental para a manutenção de relacionamentos entre adultos (Hinde, 1997). Face ao fato destes amigos estarem ligados a diferentes países, também se investigou como essa amizade teve início e os interesses compartilhados, apesar das possíveis diferenças culturais. Diante de fatores como diferenças culturais e distância, que poderiam dificultar o estabelecimento e manutenção destas amizades, foram investigadas as dificuldades nas amizades internacionais. Finalmente, devido ao fato de o amigo ser de outro país, também se verificou se a atração por um amigo estrangeiro também indicaria alguma atração pelo país desse amigo. Os dados sobre a rede internacional de amigos (nacionalidade e idioma de comunicação com cada amigo) foram provenientes das tabelas preenchidas com esses dados. Os meios de comunicação empregados com esses amigos foram investigados por meio de uma questão fechada, assim como a forma como a amizade teve início e interesses comuns. Caso as alternativas propostas nas questões fechadas não representassem o caso de cada participante, estes podiam indicar outras opções, que também foram incluídas nos resultados.

\section{A rede internacional de amigos}

Os 120 participantes mencionaram de um a cinco amigos estrangeiros, totalizando 331 amigos provenientes de 59 países. A maioria (42 participantes) indicou apenas um amigo estrangeiro. Vinte indicaram dois amigos, 14 indicaram três, 13 mencionaram quatro e 31 citaram cinco amigos, que era o limite proposto no instrumento de coleta de dados. A Tabela 1 indica a origem dos amigos:

Uma divisão por continentes resulta em 116 amigos da América do Norte, 119 da Europa, 40 da América do Sul, 24 da África, 19 da Ásia, 11 da Oceania e dois da América Central. Em 12 casos, esses amigos estavam vivendo em outros países. Quatro estavam nos EUA (do Senegal, Nova Zelândia, Peru e México), um nas Filipinas (dos EUA), um na Suécia (da República Tcheca), um na Inglaterra (da Austrália), um na Indonésia (dos EUA), um na China (dos EUA), um na Espanha (da Inglaterra), um na Rússia (da Austrália) e um na Escócia (da Irlanda).

\section{A comunicação com amigos internacionais}

Dois aspectos foram investigados quanto à comunicação com amigos internacionais: os meios de comunicação e os idiomas utilizados na comunicação, ambos a partir de questões fechadas. Em ambos os casos, mais de uma opção poderia ser citada. Os principais meios de comunicação foram a Internet, em 227 amizades, o contato pessoal (91 amizades), o telefone (61) e as cartas (12).

O principal idioma de comunicação foi o Inglês, utilizado em 199 amizades, seguido pelo Português (113 amizades), Espanhol (34), Francês (11) e Alemão (10). O Crioulo, Italiano e Russo foram utilizados em duas amizades cada e o idioma holandês em apenas um relacionamento. Em alguns casos dois idiomas eram utilizados: Português e Inglês (27 amizades), Português e Espanhol (15), Português e Francês (3), Português e Crioulo (2), Inglês e Francês (3), Inglês e Espanhol (2), Inglês e Alemão (1). Raramente eram utilizados três idiomas: Português, Espanhol e Alemão (1), Português, Inglês e Russo (2) e Português, Inglês e Espanhol (1).

\section{Início da amizade e interesses comuns}

$\mathrm{O}$ início da amizade e os interesses compartilhados foram investigados por meio de questões fechadas, contudo, os participantes podiam indicar outra resposta, caso julgassem necessário. A forma como se deu o início da amizade foi indicada em 313 relacionamentos. Em 286 casos, o início foi por contato pessoal. Este se deu no exterior (147 casos) ou no Brasil (139). $\mathrm{O}$ início da amizade por meio de um contato online deu-se em 
Tabela 1.

\begin{tabular}{c|l|c}
\multicolumn{1}{c}{ A origem dos amigos por país } & Total \\
\hline $\begin{array}{c}\text { Número de } \\
\text { Amigos }\end{array}$ & & \multicolumn{1}{c}{ País(es) de Origem } \\
\hline 91 & EUA & 91 \\
26 & Alemanha & 26 \\
13 & México & 13 \\
12 & Argentina, Canadá e França & 36 \\
11 & Holanda & 11 \\
10 & Espanha & 10 \\
9 & Peru e Portugal & 18 \\
8 & Áustria e Itália & 16 \\
6 & Cabo Verde, Colômbia, Inglaterra e Nova Zelândia & 24 \\
5 & Austrália, Guiné Bissau e Japão & 15 \\
4 & Chile, China e Equador & 12 \\
3 & Costa do Marfim, Escócia, Índia, Irlanda e República Tcheca & 15 \\
2 & África (sem especificar o país), Angola, Coréia, Eslováquia, Honduras, & 24 \\
1 & Noruega, Rússia, Sérvia, Suécia, Tailândia, Ucrânia e Venezuela & \\
& África do Sul, Bélgica, Benin, Bolívia, Dinamarca, Eslovênia, Filipinas, & 20 \\
\hline Total & Kuwait, Lituânia, Macedônia, Moçambique, Malásia, Paraguai, Polônia, & \\
\hline & Senegal, Serra Leoa, Suíça, Tunísia, Turquia e Uruguai & 331 \\
\hline
\end{tabular}

24 casos. Em dois casos, o primeiro contato foi por telefone e houve um caso do contato inicial por cartas. Essas amizades apresentavam diversos interesses em comum, sendo que os participantes podiam indicar mais de um interesse a partir de uma lista com 10 opões, incluindo lazer (96 amizades), música (67), turismo (62), cinema (57), esportes (40), assuntos ligados à família (36), artes e ciências (25 cada), literatura e religião (24 cada). Outros ainda foram citados, como política (5), assuntos amorosos (4), culinária (3), cultura (2), trabalho (2), universidade ou estudos (2), psicologia (2), além de pesquisa em história, baladas, humor, moda, atividades de cunho social (ONGs), gastronomia, liderança, internacionalismo, fluência em língua estrangeira, economia, danças de salão, fotografia, ciências humanas, história, confidências, ideias e pensamentos, sexo, filosofia e assuntos pessoais.

\section{Dificuldades nas amizades internacionais}

Aos 120 participantes responderam a uma questão aberta sobre as dificuldades percebidas em suas amizades internacionais, cujo conteúdo foi analisado e organizado em categorias. Apenas seis participantes não citaram nenhuma dificuldade em suas amizades com estrangeiros. Os demais citaram diferentes dificuldades, relacionadas principalmente com ausência física ou distância (67 participantes) e diferenças culturais, incluindo a diferença de idioma (23 participantes).

Primeiramente, os participantes consideraram que a ausência física e a distância exerciam efeitos negativos sobre a amizade de modo geral, como nos seguintes casos: a distância "compromete seriamente a amizade" (38); a distância "dificulta o aprofundamento da amizade" (83), a ausência afeta a "intensidade da amizade " (98) e "dificulta uma amizade mais sólida" (108). Ainda é "difícil manter amizade sem presença física e condições de comunicação mais eficientes" (119). A ausência física também afeta propriedades da amizade, como a intimidade: "difícil manter um vínculo forte conversando só por e-mail e telefone. A intimidade é prejudicada" (33).

De modo mais específico, a ausência física e a distância foram consideradas como fatores prejudiciais ao convívio, pois impedem "estar convivendo mais a fundo com o amigo" (27) e o "convívio constante e em um mesmo ambiente ou grupo de pessoas" (14). Outras respostas indicaram não haver mais "convívio físico e passei pouco tempo por lá" (16), apontaram a "escassez de oportunidades para estar junto" (104) e a falta sentida da presença do amigo: "sinto muito falta de seu abraço, risadas e esporros” (105). A ausência física e a distância também prejudicam o compartilhamento de atividades, as manifestações de afeto e apoio e a comunicação não verbal: "deixamos de fazer diversas coisas juntos" (49), "é difícil o encontro pessoal e marcar coisas em comum" (4); por "não ter por perto, ajudando e compartilhando a vida uma da outra" (20); "o mais difícil é estar longe de quem você gosta, não podendo passear, abraçar..." (49); "todo mundo um dia quer ser lamentar e chorar no ombro do amigo, mas a distância impede" (51); "a presença física na amizade é muito importante, às vezes dá para ver como a pessoa está só de olhar para ela" (54). Mesmo a expectativa de separação é vista como um problema: "saber que vai embora em breve e por isso tenta não se apegar demais às pessoas para não sofrer com a separação" (15).

Apesar da comunicação se manter mesmo à distância, ela é insuficiente devido à falta do convívio ou contato pessoal: "apenas Internet e telefone, nem sempre quando tenho urgência em falar com ele consigo" (44), "apesar de falar pela Internet pelo menos uma vez por semana, por telefone, ter amigo ao lado é bem diferente" (47), "por mais que a gente converse muito via Internet, saudade de tê-la por perto" (67), "mesmo que possamos nos comunicar bastante via MSN, e-mail ou Orkut" sente a falta do amigo (97), "na Internet acaba sendo um contato mais frio" (99). Neste caso, os meios de comunicação não conseguem substituir a presença física do amigo.

A ausência física e a distância geram a desinformação sobre o amigo: "não sabemos tanto da vida uma da outra" (8); "difícil acompanhar o que acontece com uma e outra" (17) ou "na vida uma da outra" (115) e "fico meio desatualizada" (53). Ainda prejudicam a comunicação entre os amigos, reduzindo a frequência ou o tempo em contato e ainda há "custo" (117). A comunicação à distância é prejudicada pela falta de tempo (3, 
$28,79,98,117)$, por ser difícil manter contato ou um contato mais frequente $(19,32,35,44,91,94,109,120)$, chegando a “deixar de se comunicar" (82). A diferença de fuso horário é outro embaraço para a comunicação $(31,35,47,73)$. Ainda há as dificuldades com os meios de comunicação, pelo uso de “outro programa de computador" (31), "já que não gosto muito de computador é difícil a gente falar constantemente" (53), "não uso tanto a Internet, por isso, o contato é bem limitado" (61).

Um segundo grupo de dificuldades encontradas nas amizades internacionais são as diferenças culturais, por vezes indicadas de modo geral sem maiores especificações $(25,41,43$, $70,81)$, relacionadas a diferentes hábitos ou costumes culturais ou a diferentes idiomas. As relacionadas a hábitos e costumes diferentes incluíram a pontualidade (10); "hábitos diferentes" (66); "hábitos típicos diferentes, formas de comer, vestir, estranhos para homens brasileiros" (21), "diferentes comidas e lugares que pode frequentar" (23), "música e comida" (106), "algumas atividades de lazer minhas não são compatíveis com as dela" (45), "algumas situações constrangedoras pelas diferenças de costumes" (89), "tenho costumes de minha região e ele da dele" (93). As diferenças culturais ainda estavam presentes no conhecimento prévio necessário para a compreensão mútua: “o que ele falava pressupunha que eu tivesse vivência cultural" (66). Finalmente, as diferenças culturais foram vistas como impedindo "grande intimidade ou proximidade entre nós" (88).

Entre as diferenças culturais, a diferença de idioma foi uma dificuldade apontada por 23 participantes. Entre as dificuldades apontadas estavam: "falar inglês o tempo todo" (2), ter "vergonha de falar espanhol e errar" (11), "o meu inglês não é muito bom" (24), "minhas limitações com o inglês" (89), "sotaque muito forte" (55), "meu inglês é bem básico, não consigo expressar o que queria" (59), além das dificuldades com a gíria $(106,114)$. Em vários casos, o idioma foi apontado como uma dificuldade maior no início do relacionamento $(58,62,74,96)$ : "no início, a língua era uma barreira. Ele aprendeu português bem em seis meses" (76), "depois dela aprender português, a amizade ficou mais próxima" (48), "no começo foi difícil compreendê-lo mas com o tempo..." (52). Finalmente, alguns indicaram as diferenças culturais como "favorecendo" as amizades (48), "bem interessantes" (65) ou "oportunidades para conhecer novas culturas" (112).

Um terceiro grupo de fatores está ligado ao conteúdo do relacionamento, especialmente a falta de intimidade e compromisso, sem indicação clara de sua origem: "as conversas com meu amigo estrangeiro não envolvem assuntos pessoais" (77), há "superficialidade" (84), "perda de intimidade" (85) ou falta de compromisso: "não temos ligação que tenha intenção de tornar a amizade duradoura" (113). Nestes casos, os participantes não identificaram se a falta de intimidade ou compromisso era devida à distância, às diferenças culturais ou outros motivos.

Um quarto grupo inclui dificuldades atribuídas ao próprio amigo, como ser "pouco comunicativo" (22), ser "ciumenta" (36), ser "fechado, tímido" (37), ter "gostos diferentes" (39), "gênio dele" (65), "temperamento" (96), "vida tomar rumos diferentes" (43), falta de "mais coisas em comum" (50) ou "dormir muito cedo e geralmente quando saímos não podemos chegar tarde" (111). Por vezes, essas diferenças individuais são atribuídas a características culturais: "típica jovem americana, pra frente, atirada, meio liberal, meio precoce" (60).

Em suma, as dificuldades apontadas estão ligadas basicamente à ausência do amigo e às limitações dos meios de comunicação para manter um relacionamento como o presencial. As diferenças culturais também foram citadas, mas a principal é o idioma. Em vários casos, há evidência de superação desta dificuldade.

\section{A relação com o país do amigo}

Uma relação de amizade entre duas pessoas não se limita ao que ocorre na díade, mas está vinculada também ao ambiente mais amplo. No caso das amizades internacionais, procurouse investigar qual a ligação ou interesse dos universitários brasileiros em relação ao país do amigo estrangeiro. A relação com o país do amigo foi avaliada por meio de uma questão fechada sobre as intenções de cada participante em quatro níveis diferentes. Em suma, 90 participantes manifestaram o desejo de visitar o país do amigo como turista, 50 tinham interesse em estudar no país do amigo, 40 gostariam de trabalhar no país do amigo e 26 residiriam no país do amigo. Apenas sete não demonstraram interesse no país do amigo.

\section{Discussão}

Apesar de ampla, a rede internacional de amigos está distribuída de forma bastante desigual, com o predomínio de alguns países, especialmente dos EUA. Quanto aos continentes, América do Norte e Europa Ocidental reúnem o maior número de amigos. Possivelmente, uma maior proximidade entre o Brasil, América do Norte e Europa Ocidental, principalmente em termos culturais, possa explicar essa tendência. Tomando-se Hinde (1997) como referencial teórico pode-se dizer que relações em um nível de complexidade (plano individual) afetam e são afetadas por relações em outros níveis de complexidade (como no plano nacional). A dependência mútua entre esses dois níveis também está implícita em de Zafra (1953).

Ainda que a Internet represente o principal meio de comunicação empregado entre amigos, o início das amizades ainda depende do contato pessoal, no Brasil ou no exterior. Também com base nas propostas de Hinde (1997), pode-se supor que a possibilidade de comunicação não é o único fator afetando o início e a manutenção da amizade. Possivelmente a amizade dependa da influência do ambiente e do grupo social para seu início. Os dados sugerem que as amizades dependem fortemente do convívio com o outro e o contato pelos meios de comunicação disponíveis não substitui o contato pessoal.

A cultura está na base de diversos interesses compartilhados, como artes, música, cinema e literatura. Contudo, o interesse parece estar mais voltado para a cultura como lazer e não como objeto de pesquisa. O compartilhamento do interesse por pesquisa ou ciência é pouco expressivo, ficando abaixo de outras atividades. Possivelmente, o primeiro passo para uma maior cooperação cultural e científica entre universitários de países diferentes poderia ocorrer em torno desses interesses compartilhados.

As dificuldades nas amizades internacionais giram em 
torno da distância e das diferenças culturais, principalmente a diferença de idioma. Estas dificuldades, contudo, são passíveis de superação, conforme explicitado em várias afirmações de problemas iniciais com o idioma que foram resolvidos. Não houve uma importância maior de outras diferenças culturais como prejudiciais para a amizade. Em algumas respostas, estas diferenças foram consideradas como impulsionadoras dessas amizades e não como restritivas. Enquanto a literatura destaca as limitações e dificuldades para estabelecer amizades com pessoas do país anfitrião entre estudantes internacionais e a tendência a fazer amizades com conterrâneos (Bailey, 2006; Brown, 2009a; Cushner \& Karim, 2004; Pandian, 2008; Sawir et al., 2008; UKCOSA, 2004), no presente trabalho as limitações e dificuldades são para manter essas amizades.

Com base em uma abordagem mais ampla dos relacionamentos, como proposta por Hinde (1997), é possível observar que nem os interesses comuns nem as dificuldades são fenômenos estritamente diádicos. Nesta pesquisa, o ambiente (espaço) e as estruturas socioculturais estavam na base das principais dificuldades dessas amizades.

A abordagem adotada neste trabalho entende que o relacionamento interpessoal não se restringe a uma díade de amigos. Isto pode ser indicado pela relação de cada universitário com o país do melhor amigo estrangeiro. $\mathrm{O}$ interesse pelo amigo tende a associar-se ao interesse em visitar, estudar, trabalhar e mesmo morar no país do amigo.

Pouco se sabe sobre as redes internacionais de amizade. A literatura tem se voltado para as dificuldades para fazer amigos com estudantes no exterior (Bektas, 2008; Ward \& Margoret, 2004), ou com estudantes locais em relação à presença de estudantes estrangeiros (e.g. Ward, 2001). Este estudo investigou, assim, as amizades internacionais de uma perspectiva mais abrangente, incluindo amigos vivendo no exterior, resultantes, na maior das vezes, de contato pessoal no Brasil ou no exterior. Quanto ao conteúdo, as amizades internacionais apresentam limitações na intimidade e compromisso, dificultando o aprofundamento de amizades internacionais, o que já havia sido observado por Chen (2006). Estas limitações na intimidade também representam um importante ponto de reflexão frente a visões clássicas da amizade, como é o caso de Aristóteles. De acordo com Rocha (2006), a convivência e "viver na intimidade" seriam indispensáveis para a amizade plena, segundo Aristóteles.

As diferenças culturais foram consideradas como dificuldades nas amizades internacionais, em concordância com Gareis (2000). Contudo, também foram apontadas como positivas, o que já havia sido sugerido por Kudo e Simkin (2003). As diferenças culturais, contudo, não foram mencionadas como empecilhos intransponíveis para a comunicação e a amizade, geralmente sendo contornadas. Como discutido por Lee (2006), há exploração das diferenças de culturas e de idiomas como aspectos específicos de relacionamentos interculturais.

As propostas de Hinde (1997) em relação ao relacionamento interpessoal apresentam-se como uma possibilidade para se analisar as amizades internacionais. $\mathrm{O}$ autor apresenta um esquema conceitual sobre as relações dialéticas entre relacionamentos e outros níveis de complexidade. Tomando-se
Hinde (1997) como referência, estas amizades (relacionamentos) envolvem o ambiente como uma dimensão importante (especialmente, a distância e a distribuição dos amigos no espaço) e as estruturas socioculturais que afetam ou enfraquecem algumas dimensões internas como a intimidade, mas não inviabilizam essas amizades.

Do ponto de vista empírico, o presente trabalho apontou características das amizades de universitários brasileiros revelando a extensão e o conteúdo dessas amizades. Do ponto de vista teórico, o trabalho procurou lidar com aspectos internos e externos da amizade, que estiveram presentes no conjunto das respostas, utilizando algumas propostas de Hinde que servem de base para a discussão.

Da perspectiva de internacionalização do saber entre pessoas de diferentes países, os dados indicam uma ampla rede de contatos formando uma rede social mediada por atividades culturais voltadas para o lazer, mas o interesse compartilhado pela ciência e pesquisa também foi observado. Possivelmente, esses aspectos compartilhados da cultura poderiam servir de base para a expansão da cooperação cultural e científica internacional.

Pode-se concluir que os universitários brasileiros estão envolvidos com amizades internacionais que dependem em grande parte do contato pessoal para existir, tendo como dificuldades principais a distância ou ausência física. $\mathrm{O}$ compartilhamento de interesses em diversos segmentos da cultura indica o potencial para o desenvolvimento de uma cooperação cultural e científica mais ampla a partir dessas relações informais.

\section{Referências}

Alfantookh, A., \& Bakry, S.H. (2008). Views on the globalisation of higher education. International Journal of Arab Culture, Management and Sustainable Development, 1(1), 123-129.

Andrade, A.M.J., \& Teixeira, M.A.P. (2009). Adaptação à universidade de estudantes internacionais: um estudo com alunos de um programa de convênio. Revista Brasileira de Orientação Profissional, 10(1), 33-44.

Bailey, C. (2006). Supporting international students in UK Higher Education: key issues, and recommendations for further research. Wolverhampton: University of Wolverhampton.

Bardin, L. (1977). Análise de Conteúdo. Lisboa: Edições 70.

Bektas, D.Y. (2008). Counselling international students in Turkish universities: current status and recommendations. International Journal for the Advancement of Counseling, 30(4), 268-278.

Brown, L. (2008). Language and anxiety: an ethnographic study of international postgraduate students. Evaluation and Research in Education, 2(3), 75-95.

Brown, L. (2009a). An ethnographic study of the friendship patterns of international students in England: an attempt to recreate home through conational interaction. International Journal of Educational Research, 48(3), 184-193.

Brown, L. (2009b). International education: a force for peace and cross-cultural understanding? Journal of Peace Education, 6(2), 209-224.

Chen, Y.W. (2006). Intercultural friendship from perspectives of East Asian students. China Media Research, 2(3), 43-58.

Cushner, K., \& Karim, A.U. (2004). Study abroad at university level. In: D. Landis, J.M. Bennett \& M.J. Bennett (Orgs), Handbook for Intercultural Training (pp.147-165). Thousand Oaks: Sage.

De Zafra Jr., C. (1953). Our pupils can build millions of international friendships. The Clearing House, 27(5), 305-307. 
Desidério, E. J. (2006). Migração internacional com fins de estudo: o caso dos africanos do programa estudante-convênio de graduação em três universidades públicas no Rio de Janeiro. (Dissertação de mestrado não publicada). ENCE/Escola Nacional de Ciências Estatísticas, Rio de Janeiro.

Gareis, E. (2000). Intercultural friendship: five case studies of German students in the USA. Journal of Intercultural Studies, 21(1), 67-91.

Hinde, R. A. (1997). Relationships: a dialectical perspective. Hove: Psychology Press.

Kreber, C. (2009). Different perspectives on internationalization in higher education. In C. Kreber (Org.), Internationalizing the curriculum in Higher Education (pp. 1-14). San Francisco: Jossey-Bass.

Kudo, K., \& Simkin, K. A. (2003). Intercultural friendship formation: the case for Japanese students at an Australian university. Journal of Intercultural Studies, 24, 91-114.

Lee, P.W. (2006). Bridging cultures: understanding the construction of relational identity in intercultural friendships. Journal of Intercultural Communication Research, 35(1), 3-22.

Maundeni, T. (2001). The Role of social networks in the adjustment of African students to British society: Students' perception. Race, Ethnicity and Education, 4(3), 253-276.

Organização das Nações Unidas (1948). Declaração Universal dos Direitos do Homem. Genebra: ONU.
Ortega, F. (2002). Genealogias da Amizade. São Paulo: Iluminuras.

Pandian, A. (2008). Multiculturalism in higher education: a case study of middle eastern students' perceptions and experiences in a Malaysian university. International Journal of Asia Pacific Studies, 4(1), 33-59.

Rocha, Z. (2006). O amigo, um outro si mesmo: A philia na metafísica de Platão e na ética de Aristóteles. Psyché, 10(17), 65-86.

Sawir, E., Marginson, S., Deumert, A., Nyland, C., \& Ramia, G. (2008). Loneliness and international students: an Australian study. Journal of Studies in International Education, 12(2), 148-180.

Subuhana, C. (2009). A experiência sociocultural de universitários da África Lusófona no Brasil: entremeando histórias. Pro-Posições, 20(1), 103-126.

UKCOSA (2004). Broadening our horizons. Londres: UKCOSA.

Ward, C., \& Masgoret, A.M. (2004). The experiences of international students in New Zealand: a report on the national survey. Wellington: Ministry of Education.

Ward, C. (2001). The impact of international students on domestic students and host institutions. Wellington: New Zealand Ministry of Education.

Ward, C., Bochner, S., \& Furnham, A. (2001). The psychology of culture shock. Londres: Routledge.

Zhao, M., \& Wildemeersch, D. (2008). Hosting foreign students in European universities: international and intercultural perspectives. European Education, 40(1), 51-62.

Agnaldo Garcia, pós-doutor e doutor em Psicologia Experimental pela Universidade de São Paulo, é professor associado na Universidade Federal do Espírito Santo. Endereço para correspondência: Av. Fernando Ferrari, 514, Goiabeiras, Vitória - ES. CEP 29075-910. Telefone/fax: (27)4009-2501. E-mail: agnaldo.garcia@uol. com.br 